\title{
THE ARF AND SATO LINK CONCORDANCE INVARIANTS
}

\author{
RACHEL STURM BEISS
}

\begin{abstract}
The Kervaire-Arf invariant is a $Z / 2$ valued concordance invariant of knots and proper links. The $\beta$ invariant (or Sato's invariant) is a $Z$ valued concordance invariant of two component links of linking number zero discovered by J. Levine and studied by Sato, Cochran, and Daniel Ruberman. Cochran has found a sequence of invariants $\left\{\beta_{i}\right\}$ associated with a two component link of linking number zero where each $\beta_{i}$ is a $Z$ valued concordance invariant and $\beta_{0}=\beta$. In this paper we demonstrate a formula for the Arf invariant of a two component link $L=X \cup Y$ of linking number zero in terms of the $\beta$ invariant of the link:
\end{abstract}

$$
\operatorname{arf}(X \cup Y)=\operatorname{arf}(X)+\operatorname{arf}(Y)+\beta(X \cup Y) \quad(\bmod 2) .
$$

This leads to the result that the Arf invariant of a link of linking number zero is independent of the orientation of the link's components. We then establish a formula for $|\beta|$ in terms of the link's Alexander polynomial $\Delta(x, y)=$ $(x-1)(y-1) f(x, y)$ :

$$
|\beta(L)|=|f(1,1)| .
$$

Finally we find a relationship between the $\beta_{i}$ invariants and linking numbers of lifts of $X$ and $Y$ in a $Z / 2$ cover of the compliment of $X \cup Y$.

\section{INTRODUCTION}

The Kervaire-Arf invariant [KM, R] is a $Z / 2$ valued concordance invariant of knots and proper links. The $\beta$ invariant (or Sato's invariant) is a $Z$ valued concordance invariant of two component links of linking number zero discovered by Levine (unpublished) and studied by Sato [S], Cochran [C], and Daniel Ruberman. Cochran [C] has found a sequence of invariants $\left\{\beta_{i}\right\}$ associated with a two component link of linking number zero where each $\beta_{i}$ is a $Z$ valued concordance invariant and $\beta_{0}=\beta .23$ Theorem 1 demonstrates a formula for the Arf invariant of a two component link $L=X \cup Y$ of linking number zero in terms of the $\beta$ invariant of the link:

Theorem 1. $\operatorname{arf}(X \cup Y)=\operatorname{arf}(X)+\operatorname{arf}(Y)+\beta(X \cup Y)(\bmod 2)$.

This leads to the result that the Arf invariant of a link of linking number zero is independent of the orientation of the link's components. In Theorem 2 we establish a formula for $|\beta|$ in terms of the link's Alexander polynomial $\Delta(x, y)=(x-1)(y-1) f(x, y)$.

Received by the editors December 1, 1988.

1980 Mathematics Subject Classification (1985 Revision). Primary 57M25. 
Theorem 2. $|\beta(L)|=|f(1,1)|$.

Finally, in Theorem 3 we find a relationship between the invariants and linking numbers of lifts of $X$ and $Y$ in a $Z / 2$ cover of the compliment of $X \cup Y$ :

Theorem 3. $-\frac{1}{2} \operatorname{lk}\left(Y^{0}, Y^{1}\right)=\sum_{j=0}^{N-1} 4^{j} \beta_{j}(L)-2(4)^{N-1} \operatorname{lk}\left(c_{N}^{0}, c_{N}^{1}\right)$.

Acknowledgment. This paper is based on my thesis prepared at the Courant Institute of Mathematical Sciences. I would like to thank Professor Sylvain Cappell, my advisor, for much help and encouragement and Daniel Ruberman for many helpful discussions.

We will begin by recalling a few definitions.

Let $V$ be a vector space of dimension $2 n$ over $Z / 2$. Let $\varphi: V \times V \rightarrow Z / 2$ be a nonsingular symmetric bilinear form such that $\varphi(a, a)=0$ for every $a$ in $V$. The map $q: V \rightarrow Z / 2$ is a quadratic form with respect to $\varphi$ if and only if

$$
q(a+b)=q(a)+q(b)+\varphi(a, b) \text { for every } a, b \text { in } V .
$$

Definition 1.1. The set $\left\{a_{1}, b_{1}, \ldots, a_{n}, b_{n}\right\}$ is a symplectic basis of $V$ with respect to $\varphi$ if it is a basis of $V$ and if $\varphi\left(a_{i}, b_{j}\right)=\delta_{i j}$ and $\varphi\left(a_{i}, a_{j}\right) \varphi\left(b_{i}, b_{j}\right)=$ 0 .

Definition 1.2 (Arf invariant of a quadratic form). Let $\left\{a_{1}, b_{1}, \ldots, a_{n}, b_{n}\right\}$ be a symplectic basis of $V$ with respect to $\varphi$. Then

$$
\operatorname{arf}(q)=\sum_{i=1}^{n} q\left(a_{i}\right) q\left(b_{i}\right)
$$

is the Arf invariant of $q$.

The above definition is independent of the choice of symplectic basis (see Arf $[\mathrm{A}])$.

Let $K$ be a knot in $S^{3}$ and $M$ an oriented Seifert surface spanning $K$. Then $H_{1}(M ; Z / 2)$ is a vector field over $Z / 2$ of dimension $2 q$ where $q=$ genus of $M$. We have a symmetric nonsingular bilinear form

$$
\text { int }_{2}: H_{1}(M ; Z / 2) \times H_{1}(M ; Z / 2) \rightarrow Z / 2
$$

defined by $\operatorname{int}_{2}(\underline{a}, \underline{b})=$ the $\bmod 2$ intersection number of cycles $a, b$ which represent $\underline{a}$ and $\underline{b}$. Since $M$ is orientable, $\operatorname{int}_{2}(\underline{a}, \underline{a})=0$ for every $\underline{a}$ in $H_{1}(M ; Z / 2)$.

From now on we will assume we have fixed an orientation of $S^{3}$. Since $M$ and $S^{3}$ are now oriented we can distinguish between the positive and negative normal directions to $M$.

Given $\underline{a}$, an element of $H_{1}(M)$, let $a$ be a curve in $M$ which represents $\underline{a}$ and $a^{+}, a^{-}$the push offs of $a$ in the positive and negative normal directions to $M$. Define $\delta_{2}: H_{1}(M ; Z / 2) \rightarrow Z / 2$ by $\delta_{2}(\underline{a})=1 \mathrm{k}\left(a, a^{+}\right) \bmod 2$ the modulo 
two linking number between $a$ and $a^{+}$. We have

$$
\delta_{2}(\underline{a}+\underline{b})=\delta_{2}(\underline{a})+\delta_{2}(\underline{b})+\operatorname{int}_{2}(\underline{a}, \underline{b})
$$

$[\mathrm{KM}]$, so $\delta_{2}$ is a quadratic form associated with int ${ }_{2}$.

Definition 1.3 (Arf invariant of a knot). The Arf invariant of a knot $K$ is equal to the Arf invariant of the quadratic form $\delta_{2}$.

Let $L=X \cup Y$ be a two component link with even linking number in $S^{3}$ whose components $X$ and $Y$ are oriented. Let $M$ be a connected Seifert surface for $L$. Then $H_{1}(M ; Z / 2)$ is a vector space over $Z / 2$ of dimension $2 g+1$ where $g=$ genus of $M$. The modulo two intersection form is nonsingular on the quotient space of $H_{1}(M ; Z / 2)$ given by $H_{1}(M ; Z / 2) / J$, where $J$ is a one-dimensional subspace generated by one boundary component of $M$.

Definition 1.4 (Arf invariant of a link). The Arf invariant of link $L$ is equal to the Arf invariant of the quadratic form $\delta_{2}$ restricted to the quotient space $H_{1}(M ; Z / 2) / J$ of $H_{1}(M ; Z / 2)$.

The above definition is well defined for links of even linking number $[R]$.

If $L=X \cup Y$ has linking number zero then there exist Seifert surfaces $M_{x}, M_{y}$ for $X$ and $Y$ such that $X \cap M_{Y}=Y \cap M_{x}=\varnothing$. Therefore $M_{x} \cap M_{y}=$ $C_{1} \cup C_{2} \cup \cdots \cup C_{n}$ where $C_{j}$ is a circle embedded in $M_{x}$ and $M_{y}$. Orient $C_{j}$ so that a positive unit tangent to $C_{j}$ together with the positive unit normals to $M_{x}$ and $M_{y}$ give the chosen orientation of $S^{3}$.

The intersection $M_{x} \cap M_{y}$ has a natural framing of its normal bundle given by the normal 1 -fields $(\vec{v}, \vec{w})$. By the Thom-Pontryagin construction $\left(M_{x} \cap\right.$ $\left.M_{y}, \vec{v}, \vec{w}\right)$ corresponds to an element of $\Pi_{3}\left(S^{2}\right)$.

Definition 1.5 (the $\beta$ or Sato invariant). $\beta(L)$ is the element of $\Pi_{3}\left(S^{2}\right)$ given by $\left(M_{x} \cap M_{y}, \vec{v}, \vec{w}\right)$.

Alternate definition to 1.5a (the $\beta$ or Sato invariant).

$$
\beta(L)=2 \sum_{i<j} 1 \mathrm{k}\left(C_{i}, C_{j}\right)+\sum_{j=1}^{n} \operatorname{lk}\left(C_{j}, C_{j}^{+}\right)
$$

where $\operatorname{lk}(a, b)$ is the linking number between $a$ and $b$ with respect to the chosen orientation of $S^{3}$.

Definitions 1.5 and 1.5a are obviously the same if $M_{x} \cap M_{y}$ is connected. In Lemma 2.1 we show that if $M_{x} \cap M_{y}$ has two or more components then $M_{x}$ and $M_{y}$ can be altered so that the altered surfaces intersect in a connected manifold. Figure 1.1 illustrates how the definitions are the same when $M_{x} \cap M_{y}=c_{1} \cup \mathcal{c}_{2}$ has two components and $M_{x}, M_{y}$ are altered so that the new intersection $c$ is connected. 

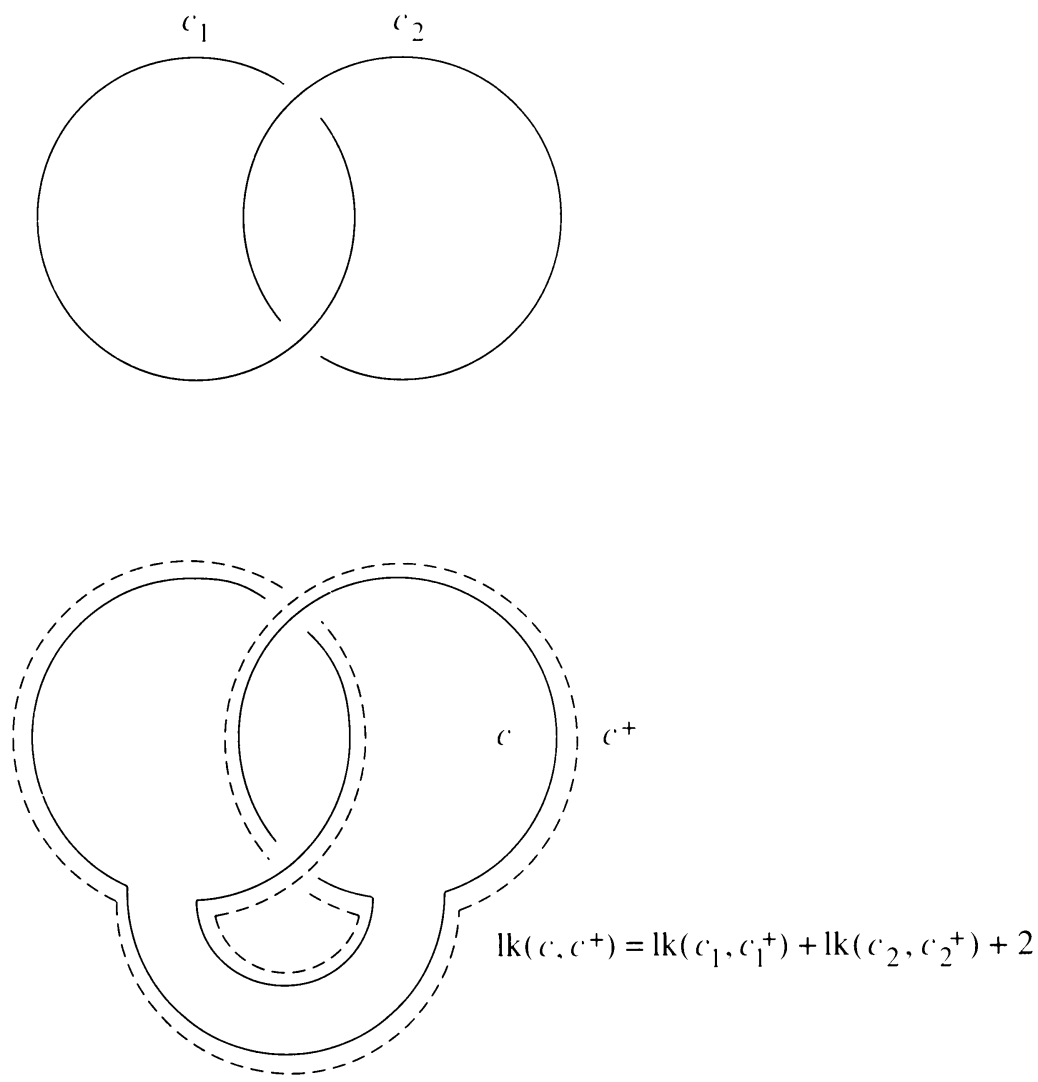

Figure 1.1

\section{A RELATIONSHip BETWEen the $\beta$ AND ARF INVARIANTS}

In this section we establish a relationship between the $\beta$ invariant and the Arf invariant of a link of linking number zero.

Theorem 1. If $L=X \cup Y$ is an oriented link of linking number zero then $\operatorname{arf}(L)=\operatorname{arf}(X)+\operatorname{arf}(Y)+\beta(L)(\bmod 2)$.

First we will find two oriented Seifert surfaces $M_{X}, M_{Y}$ for $X, Y$ respectively such that $M_{X} \cap M_{Y}$ consists of only one circle. We cut $M_{X}$ and $M_{Y}$ along this circle and reglue to obtain an oriented Seifert surface $M$ for $L$. Surface $M$ will be used to compute $\operatorname{arf}(L)$.

Lemma 2.1. Let $L=X \cup Y$ be a link of linking number zero. There exist orientable Seifert surfaces $M_{X}$ and $M_{Y}$ spanning $X$ and $Y$ such that $M_{X} \cap$ $M_{Y}=\varnothing$ or one circle. Furthermore this circle is not null homologous in $H_{1}\left(M_{X}\right)$ of $H_{1}\left(M_{Y}\right)$.

Proof. Let $N_{X}, N_{Y}$ be oriented Seifert surfaces for $X$ and $Y$ such that $N_{X} \cap$ $N_{Y}=\left\{c_{1}, \ldots, c_{n}\right\}$, where each $c_{j}$ is an embedded circle. Change circle inter- 


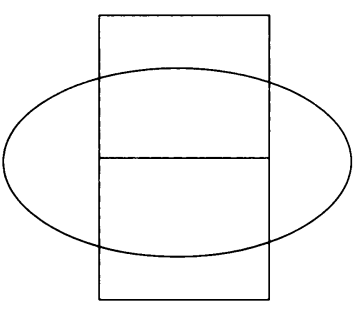

band intersection

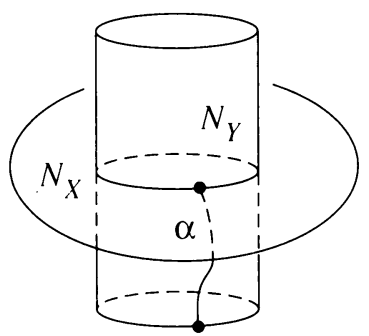

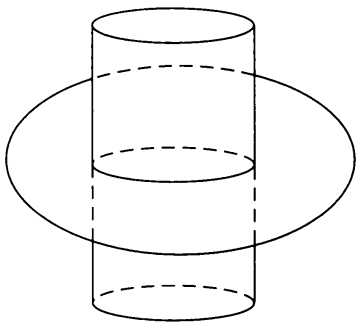

circle intersection

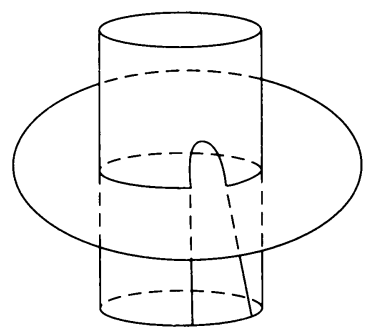

FIGURE 2.1

sections $c_{1}$ and $c_{2}$ to band intersections by the following method of Cooper [Co]. Let $\alpha_{1}, \alpha_{2}$ be paths from $\partial N_{Y}$ to points on $c_{1}$ and $c_{2}$ respectively. Push $N_{Y}$ in along $\alpha_{1}$ and $\alpha_{2}$ so $c_{1}$ and $c_{2}$ become band intersections (see Figure 2.1). Let $p_{1}$ and $p_{2}$ be two points on the resulting band intersections. Let $\gamma$ be an arc in $N_{X}$ from $p_{1}$ to $p_{2}$ and oriented from $p_{1}$ to $p_{2}$ such that the intersection number of $\gamma$ with $N_{Y}$ at $p_{1}$ is +1 and the intersection number of $\gamma$ with $N_{X}$ at $p_{2}$ is -1 (see Figure 2.2(i)). Now attach a handle to $N_{Y}$ whose core coincides with $\gamma$ (see Figure 2.2(ii)). Our two band intersections have been transformed into two different band intersections. If we attach two handles to $N_{X}$ whose cores coincide with the two $\operatorname{arcs}$ in $\partial N_{Y}=Y$ which were pushed in along paths $\alpha_{1}$ and $\alpha_{2}$ then the band intersections will become one circle intersection (see Figure 2.2(iii)).

Let us call our new surfaces $N_{X}^{\prime}$ and $N_{Y}^{\prime}$. These new surfaces intersect in $N_{X}^{\prime} \cap N_{Y}^{\prime}=\left\{a, c_{3}, \ldots, c_{n}\right\}$, where $a$ was formed from $c_{1}$ and $c_{2}$. If we repeat the above process we can find oriented surfaces $M_{X}$ and $M_{Y}$ such that $M_{X} \cap M_{Y}=$ one circle $c$.

Suppose $M_{X} \cap M_{Y}=c$ and $c$ is null homologous in $H_{1}\left(M_{X}\right)$. Then $c$ bounds a surface $N$ in $M_{X}$. Look at a neighborhood of $N$ in $S^{3}$ parametrized by $N \times[1,-1]$, where $N \times 0$ coincides with $N$. Replace $M_{X}$ with $M_{X}-N \cup$ $\partial N \times[1,0] \cup N \times 1$. Now round off the corner at $N \times 0$ and we will no longer have an intersection along $c$. 
(i)

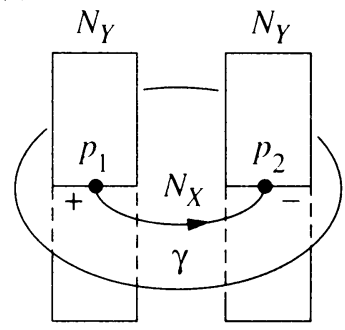

(ii)

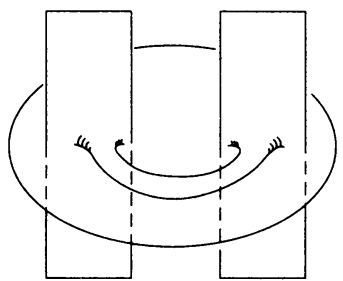

(iii)

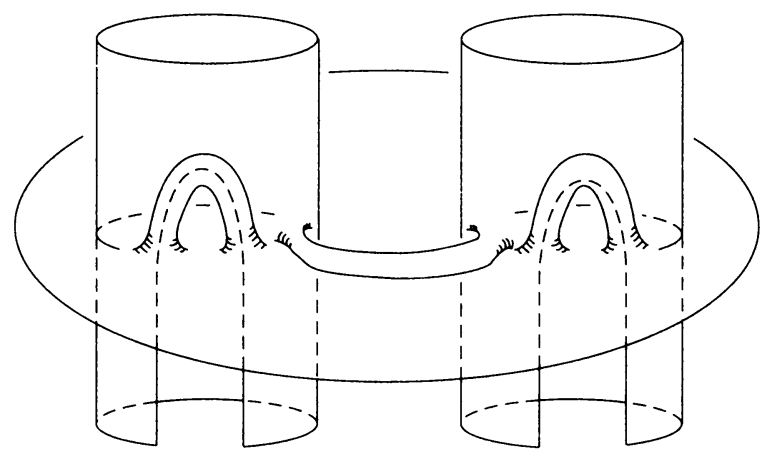

FIGURE 2.2

Proof of Theorem 1. By Lemma 2.1 we can assume we have oriented Seifert surfaces $M_{X}$ and $M_{Y}$ for $X, Y$ respectively such that $M_{X} \cap M_{Y}=\varnothing$ or $M_{X} \cap M_{Y}=c$, where $c$ is an embedded circle and $c$ not homologous to zero in $H_{1}\left(M_{X}\right)$ or $H_{1}\left(M_{Y}\right)$.

If $M_{X} \cap M_{Y}=\varnothing$ then $L$ is a boundary link. From the methods of Robertello [R] it easily follows that $\operatorname{arf}(L)=\operatorname{arf}(X)+\operatorname{arf}(Y)$. Since the $\beta$ invariant for a boundary link is zero the formula holds in this case.

Assume that $M_{X} \cap M_{Y}=c$. We can find symplectic bases :

$$
\left\{\underline{c}, \underline{d}_{1}, \underline{c}_{2}, \underline{d}_{2}, \ldots, \underline{c}_{g}, \underline{d}_{g}\right\}, \quad\left\{\underline{c}, \underline{d}_{1}^{\prime}, \underline{e}_{2}, \underline{f}_{2}, \ldots, \underline{e}_{h}, \underline{f}_{h}\right\}
$$

for $H_{1}\left(M_{X} ; Z / 2\right)$ and $H_{1}\left(M_{Y} ; Z / 2\right)$ respectively, where $g=$ genus $M_{X}$ and $h=$ genus $M_{Y}$, and $c$ represents $\underline{c}$.

We now construct $M$, an oriented Seifert surface for $L$, from $M_{X}$ and $M_{Y}$. Cut $M_{X}$ and $M_{Y}$ along $c$. Attach $M_{X}-c$ to $M_{Y}-c$ so that the positive sides of $M_{X}-c$ and $M_{Y}-c$ form the positive side of $M . M$ is orientable.

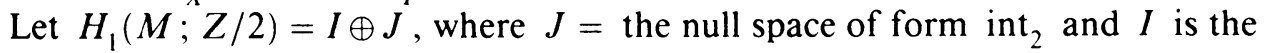
subspace with symplectic basis :

$$
\left\{\underline{c}, \underline{d}, \underline{c}_{2}, \underline{d}_{2}, \ldots, \underline{c}_{g}, \underline{d}_{g}, \underline{e}_{2}, \underline{f}_{2}, \ldots, \underline{e}_{h}, \underline{f}_{h}\right\}
$$

where $d=d_{1} \cup d_{1}^{\prime}$ represents $\underline{d}$. The self-linking numbers of the basis element of $I$ have not changed with the exception of $\underline{d}: \delta_{2}(\underline{d})=\delta_{2}\left(\underline{d}_{1}\right)+\delta_{2}\left(\underline{d}_{1}^{\prime}\right)+1$. 

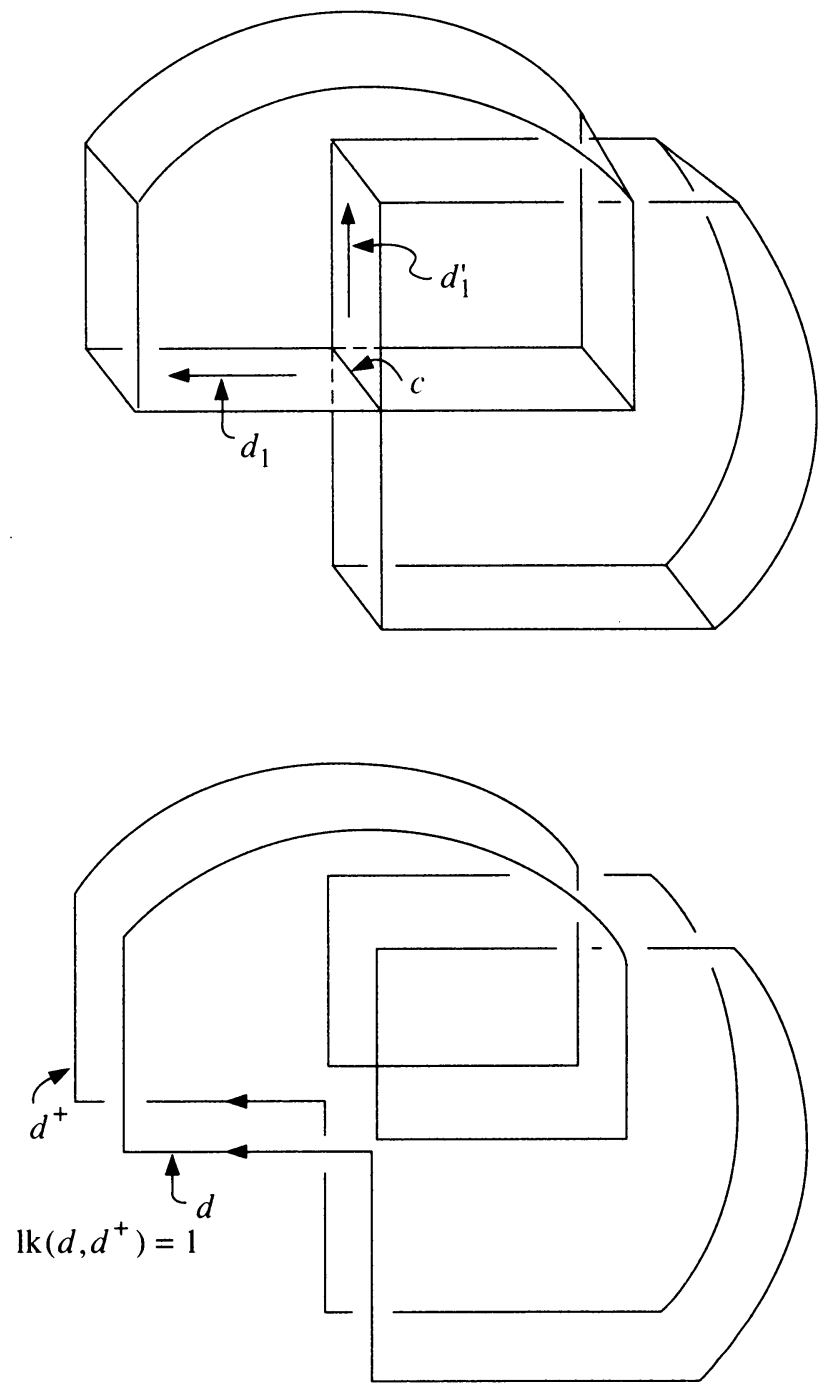

FIGURE 2.3

The extra 1 appears because $\underline{d}_{1}$ and $\underline{d}_{1}^{\prime}$ intersects at a point. Figure 2.3 is a local picture of $M_{X} \cup M_{Y}$ near $d_{1}$ and $d_{1}^{\prime}$ and shows how the extra linking number appears.

Use the above symplectic basis of $I$ to calculate $\operatorname{arf}(L)$ :

$$
\begin{aligned}
\operatorname{arf}(L) \equiv & \delta_{2}(\underline{c}) \delta_{2}(\underline{d})+\sum_{j=2}^{g} \delta_{2}\left(c_{j}\right) \delta_{2}\left(\underline{d}_{j}\right)+\sum_{j=2}^{h} \delta_{2}\left(\underline{e}_{j}\right) \delta_{2}\left(\underline{f}_{j}\right) \\
\equiv & \delta_{2}(\underline{c})\left(\delta_{2}\left(\underline{d}_{1}\right)+\delta_{2}\left(\underline{d}_{1}^{\prime}\right)+1\right) \\
& +\sum_{j=2}^{g} \delta_{2}\left(\underline{c}_{j}\right) \delta_{2}\left(\underline{d}_{j}\right)+\sum_{j=2}^{h} \delta_{2}\left(\underline{e}_{j}\right) \delta_{2}\left(\underline{f}_{j}\right) \\
\equiv & \delta_{2}(\underline{c})+\operatorname{arf}(X)+\operatorname{arf}(Y) \bmod 2
\end{aligned}
$$


and $\delta_{2}(\underline{c}) \equiv \beta(L) \quad(\bmod 2)$ so,

$$
\operatorname{arf}(L) \equiv \operatorname{arf}(X)+\operatorname{arf}(Y)+\beta(L) \quad(\bmod 2) .
$$

Corollary 1. The Arf invariant of a li,lk of linking number zero is independent of the orientation of the components of the link.

Proof. We must show that $\beta(L)(\bmod 2)$ is independent of the link's orientation.

Definition 1.5 for $\beta(L)$ shows

$$
\begin{aligned}
\beta(L) & =2 \sum_{i<j} \mathrm{lk}\left(c_{i}, c_{j}\right) L+\sum_{j=1}^{2 p} \mathrm{lk}\left(c_{j}, c_{j}^{+}\right) \\
& \equiv \sum_{j=1}^{2 p} \mathrm{lk}\left(c_{j}, c_{j}^{+}\right) \quad(\bmod 2) .
\end{aligned}
$$

Each term $\operatorname{lk}\left(c_{j}, c_{j}^{+}\right)$is independent of the orientation of the components of the link.

\section{A FORMUla FOR $|\beta(L)|$ IN TERMS OF THE \\ ALEXANDER POLYNOMIAL OF $L$}

In this section we establish a relationship between the $\beta$ invariant of a link and its Alexander polynomial $\Delta(x, y)$. If $L=X \cup Y$ is a link of linking number zero then it has an Alexander polynomial of the form

$$
\Delta(x, y)=(x-1)(y-1) f(x, y) .
$$

Theorem 2. $|\beta(L)|=|f(1,1)|$.

We compute $\Delta(x, y)$ using an algorithm by Cooper [Co] which is summarized in the following paragraph:

Let $L=X \cup Y$ be an oriented link in $S^{3}$ and $M_{X}, M_{Y}$ Seifert surfaces with orientations inherited from those of $X$ and $Y$ such that $M_{X} \cap M_{Y}$ consists of only clasp intersections (see Figure 3.1(i)). Cooper defines two bilinear forms

$$
u, v: H_{1}\left(M_{X} \cup M_{Y}\right) \times H_{1}\left(M_{X} \cup M_{Y}\right) \rightarrow Z
$$

by $u(\underline{a}, \underline{b})=\operatorname{lk}\left(a, b^{--}\right)$and $v(\underline{a}, \underline{b})=\operatorname{lk}\left(a, b^{-+}\right)$, where $a, b$ are curves in $M_{X}$ and $M_{Y}$ respectively which represent $\underline{a}$ and $\underline{b}$. The notation $\operatorname{lk}\left(a, b^{--}\right)$ stands for the linking number between $a$ and $b^{--}$where $b^{--}$is the push off of $b$ in the negative normal direction to $M_{X}$ and negative normal direction to $M_{Y}$. Similarly $\operatorname{lk}\left(a, b^{-+}\right)$is the linking number between $a$ and the push off of $b$ in the negative normal direction to $M_{X}$ and positive normal direction to $M_{Y}$. When $a$ or $b$ passes from $M_{X}$ to $M_{Y}$ it must contain the clasp intersection. Near a clasp the push off of a curve will lie in one of the four quadrants formed by the clasp intersection as suggested in Figure 3.1(ii). Cooper observed that

\footnotetext{
${ }^{1}$ The quantity $|f(1,1)|$ is equal to $\left|a_{1}\right|$, where $a_{1}$ is a Conway polynomial coefficient. In [C] the Conway polynomial coefficients are found to be related to Milnor's $\mu$-invariants and to certain linking numbers.
} 
(i) A clasp intersection

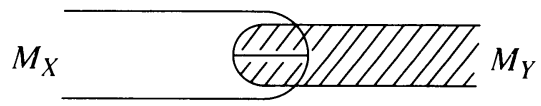

(ii)

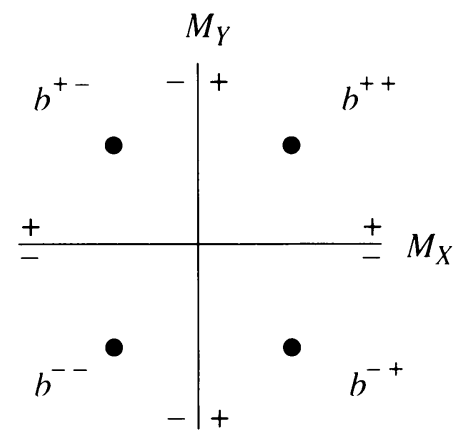

(iii) Changing a circle intersection to two clasps
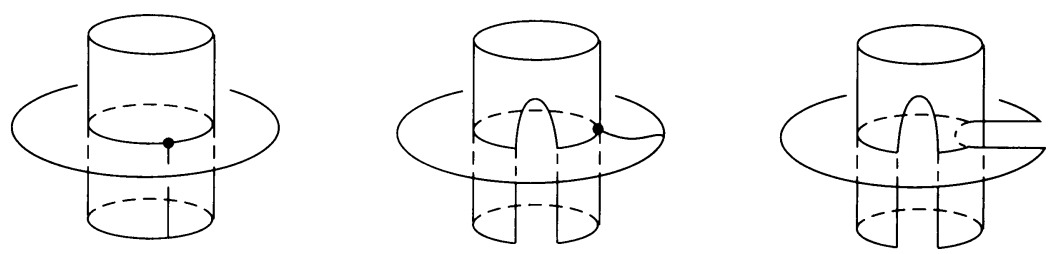

Figure 3.1

$$
H_{1}\left(M_{X} \cup M_{Y}\right) \cong H_{1}\left(M_{X}\right) \oplus H_{1}\left(M_{Y}\right) \oplus \widehat{H}_{0}\left(M_{X} \cap M_{Y}\right) .
$$

Loops circling through the clasps form a basis for the component of $H_{1}\left(M_{X} \cup M_{Y}\right)$ isomorphic to $\widehat{H}_{0}\left(M_{X} \cap M_{Y}\right)$. Let $A, B$ be matrices representing $u$ and $v$ respectively. Cooper shows that

$$
\Delta(x, y)=(x-1)^{-2 g}(y-1)^{-2 h} \operatorname{det}\left(x y A+A^{T}-x B-y B^{T}\right),
$$

where $g=$ genus of $M_{X}$ and $h=$ genus of $M_{y}$.

Proof of Theorem 2. By Lemma 2.1 we can assume that we have oriented Seifert surfaces $N_{X}^{\prime}$ and $N_{Y}^{\prime}$ for $X$ and $Y$ such that $N_{X}^{\prime} \cap N_{Y}^{\prime}$ is a single circle. The circle intersection can be changed as in [Co] to two clasp intersections (see Figure 3.1(iii)). Let $N_{X}, N_{Y}$ be the resulting Seifert surfaces for $X$ and $Y$ such that $N_{X} \cap N_{Y}$ is two clasps. If $N_{X}^{\prime} \cap N_{Y}^{\prime}=c$ then the invariant of $L$, 
$\beta(L)$, is by definition equal to $\mathrm{k}\left(c, c^{--}\right)=\mathrm{k}\left(c, c^{-+}\right)$. Let us choose a basis of $H_{1}\left(N_{X} \cup N_{Y}\right)$ consisting of a basis for $H_{1}\left(N_{X}\right)$ union a basis for $H_{1}\left(N_{Y}\right)$ union $\underline{c}$ where $c$ runs through the two clasps as above and represents $\underline{c}$. Notice that if curve $b$ lies in $M_{X}$ then $u(\underline{a}, \underline{b})=v(\underline{a}, \underline{b})=\operatorname{lk}\left(a, b^{-}\right)$where $b^{-}$is the push off of $b$ in the negative normal direction to $M_{X}$. If $A_{X}=A$ restricted to $H_{1}\left(N_{X}\right)$ and $B_{X}=B$ restricted to $H_{1}\left(N_{X}\right)$ then $A_{X}=B_{X}$. Similarly if $A_{Y}=A$ restricted to $H_{1}\left(N_{Y}\right)$ and $B_{Y}=B$ restricted to $H_{1}\left(N_{Y}\right)$ then $B_{Y}=A_{Y}^{T}$. Let $n=\beta(L)=1 \mathrm{k}\left(c, c^{--}\right)=1 \mathrm{k}\left(c, c^{-+}\right)$. Then with respect to the above basis for $H_{1}\left(N_{X} \cup N_{Y}\right), A$ and $B$ have the following form:

$$
A=\left[\begin{array}{ccc}
A_{X} & & \\
& & q_{i j} \\
& A_{y} & \\
q_{j i} & & n
\end{array}\right], \quad B=\left[\begin{array}{ccc}
A_{X} & & \\
& & q_{i j} \\
& A_{Y}^{T} & \\
q_{j i} & & n
\end{array}\right]
$$

where $q_{i j}=q_{j i}$. $A$ has matrices $A_{X}, A_{Y}$ and $(n)$ down the diagonal. Away from these submatrices $A$ has $i j$ th entry designated by $q_{i j}$. The $q_{i j}$ entries represent linking numbers between nonintersecting curves in $N_{X}$ and $N_{Y}$, thus $q_{i j}=q_{j i} . B$ has matrices $A_{X}, A_{Y}^{T}$ and $(n)$ down the diagonal. The $q_{i j}$ entries of $B$ are equal to those of $A$.

By Cooper's algorithm

$$
\begin{aligned}
\Delta(x, y)= & (x-1)^{-2 g}(y-1)^{-2 h} \operatorname{det} x y\left[\begin{array}{lll}
A_{X} & & \\
& & q_{i j} \\
q_{j i} & & n
\end{array}\right] \\
& +\left[\begin{array}{lll}
A_{X}^{T} & \\
& A_{Y}^{T} & \\
q_{i j} & n
\end{array}\right]-x\left[\begin{array}{lll}
A_{X} & & \\
& q_{j i} \\
& A_{Y}^{T} & \\
q_{j i} & & n
\end{array}\right]-y\left[\begin{array}{lll}
A_{X}^{T} & \\
& A_{Y} & q_{i j} \\
q_{j i} & n
\end{array}\right] \\
= & (x-1)^{-2 g}(y-1)^{-2 h} \\
& \cdot \operatorname{det}\left[\begin{array}{lll}
(y-1)\left(x A_{X}-A_{X}^{T}\right) & \\
(x-1)(y-1) q_{j i} & (x-1)\left(y A_{Y}-A_{Y}^{T}\right)(y-1) q_{i j}
\end{array}\right] .
\end{aligned}
$$

Now factor out $(x-1)$ from the first $2 g$ rows and $(y-1)$ from the next $2 h$ rows:

$$
=\operatorname{det}\left[\begin{array}{lrc}
x A_{X}-A_{X}^{T} & \cdots & (x-1) q \\
(y-1) q \cdots y A_{Y}-A_{Y}^{T} & (y-1) q \\
(x-1)(y-1) q & \cdots & (x-1)(y-1) n
\end{array}\right] .
$$

Factor our $(x-1)(y-1)$ from the last row:

$$
\begin{aligned}
& =(x-1)(y-1) \operatorname{det}\left[\begin{array}{lcc}
x A_{X}-A_{X}^{T} & & (x-1) q \\
(y-1) q \cdots y & A_{Y}-A_{Y}^{T} & (y-1) q \\
q & \ldots & n
\end{array}\right] \\
& =(x-1)(y-1) f(x, y) .
\end{aligned}
$$




$$
\begin{aligned}
f(1,1) & =\operatorname{det}\left[\begin{array}{cccc}
A_{X}-A_{X}^{T} & & & 0 \\
& A_{Y}-A_{Y}^{T} & & \vdots \\
q & \cdots & q & n
\end{array}\right] \\
& =\operatorname{det}\left(A_{X}-A_{X}^{T}\right) \operatorname{det}\left(A_{Y}-A_{Y}^{T}\right) n= \pm n= \pm \underline{\beta}(L)
\end{aligned}
$$

since $A_{X}-A_{X}^{T}$ and $A_{Y}-A_{Y}^{T}$ are intersection forms on $H_{1}\left(N_{X}\right)$ and $H_{1}\left(N_{Y}\right)$ and therefore have determinant \pm 1 .

\section{THE $\beta_{j}$ INVARIANTS}

If $L=X \cup Y$ is a link of linking number zero then Cochran has defined a series of integral concordance invariants $\beta_{j}$, where $\beta_{0}=\beta$. To define $\beta_{j}$ we must first define the notion of derived links of a link.

Definition 4.1 (Cochran [C]). If $L=X \cup Y$ is a link of linking number zero and $M_{X}, M_{Y}$ are Seifert surfaces spanning $X$ and $Y$ such that $M_{X} \cap M_{Y}=c_{1}$ where $c_{1}$ is an embedded circle, then $L=X \cup c_{1}$ is a first derived link of $L$. Link $L_{j}=X \cup c_{j}$ is a $j$ th derived link of $L$ if it is a first derived link of $L_{j-1}=X \cup \underline{c}_{j-1}$. (Note: Each derived link has the same first component.)

Definition 4.2 (Cochran [C]). The $\beta_{j}$ invariant of $L, j>0$, is equal to the $\beta$ invariant of a $j$ th derived link of $L$ and $\beta_{0}=\beta$.

Theorem 3. Let $L=X \cup Y$ be a link of linking number zero where $X$ is the unknot. Let $\Sigma$ be the $Z / 2$ cover of $S^{3}$ branched over $X$ and $Y^{0}, Y^{1}$ the two lifts of $Y$ in $\Sigma$. Then

$$
-\frac{1}{2} \operatorname{lk}\left(Y^{0}, Y^{1}\right)=\sum_{j=0}^{N-1} 4^{j} \beta_{j}(L)-2 \circ 4^{N-1} \operatorname{lk}\left(c_{N}^{0}, c_{N}^{1}\right),
$$

where $L_{N}=X \cup c_{N}$ is an $N$ th derived link of $L$, and $c_{N}^{0}, c_{N}^{1}$ are the two lifts of $c_{N}$ in $\Sigma$.

Corollary 3. $\frac{1}{2} \mathrm{lk}\left(Y^{0}, Y^{1}\right) \equiv \beta(L) \bmod 2$.

Proof. This is just line (1) modulo two.

Proof of Theorem 5. Let $M_{X}, M_{Y}$ be oriented Seifert surfaces for $X$ and $Y$ such that $M_{X} \cap M_{Y}=c_{1}$ where $c_{1}$ is an embedded circle. In $\Sigma, M_{Y}$ (cut along $c_{1}$ ) lifts to $M_{Y}^{0}$ and $M_{Y}^{1} ; c_{1}$ lifts to $c_{1}^{0}$ and $c_{1}^{1}$. Now,

$$
\partial M_{Y}^{0}=c_{1}^{0}-c_{1}^{1}+Y^{0} \text { and } \partial M_{Y}^{1}=c_{1}^{1}-c_{1}^{0}+Y^{1} \text {. }
$$

Curve $c_{1}$ has a neighborhood in $M_{Y}$ parametrized by $c_{1} \times[-1,1]$. We will choose $\gamma$ in $M_{Y}$ to be equal to $c_{1} \times-1$ or $c_{1} \times 1$. The two lifts of $\gamma$ will be contained in collar neighborhoods of $\partial M_{Y}^{0}$ and $\partial M_{Y}^{1}$. Choose $\gamma$ so that $\gamma^{0}$, the lift of $\gamma$ in $M_{Y}^{0}$, is contained in a neighborhood of $c_{1}^{0}$ in $M_{Y}^{0}$, then $\gamma^{1}$, the lift of $\gamma$ in $M_{Y}$ will be contained in a neighborhood of $c_{1}^{1}$ in $M_{Y}^{1}$. 


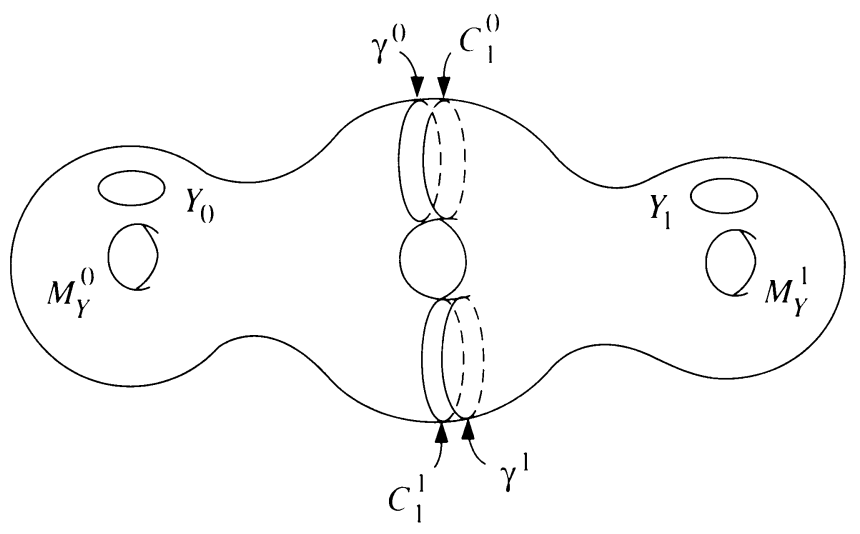

FIGURE 4.1

We would like to compute $\operatorname{lk}\left(Y^{0}, Y^{1}\right)$. First note that since $X$ is the unknot $\Sigma$ is homeomorphic to $S^{3}$ so the linking number is well defined. In $\Sigma-Y^{1}, Y^{0}$ is homologous to $c_{1}^{1}-\gamma^{0}$ and $Y^{1}$ is homologous to $c_{1}^{0}-\gamma^{1}$ (see Figure 4.1). So $\operatorname{lk}\left(Y^{0}, Y^{1}\right)=\operatorname{lk}\left(\gamma^{0}-c_{1}^{1}, Y^{1}\right)$. In $\Sigma-\left(\gamma^{0}-c_{1}^{1}\right), Y^{1}$ is homologous to $\gamma^{1}-c_{1}^{0}$, so

$$
\begin{aligned}
\operatorname{lk}\left(\gamma^{0}-c_{1}^{1}, Y^{1}\right) & =\operatorname{lk}\left(\gamma^{0}-c_{1}^{1}, \gamma^{1}-c_{1}^{0}\right) \\
& =\operatorname{lk}\left(\gamma^{0}, \gamma^{1}\right)-\operatorname{lk}\left(\gamma^{0}, c_{1}^{0}\right)-\operatorname{lk}\left(c_{1}^{1}, \gamma^{1}\right)+\operatorname{lk}\left(c_{1}^{1}, c_{1}^{0}\right) \\
& =2 \operatorname{lk}\left(c_{1}^{0}, c_{1}^{1}\right)-2 \operatorname{lk}\left(\gamma^{0}, c_{1}^{0}\right)
\end{aligned}
$$

since $\operatorname{lk}\left(\gamma^{0}, \gamma^{1}\right)=\operatorname{lk}\left(c_{1}^{0}, c_{1}^{1}\right)$ and $\operatorname{lk}\left(\gamma^{0}, c_{1}^{0}\right)=\operatorname{lk}\left(c_{1}^{1}, \gamma^{1}\right)$.

We now use a general lifting formula of linking numbers: Let $p: \widetilde{W} \rightarrow W$ be a covering space projection, and $G$ the group of covering transformations of $\widetilde{W}$. Assume linking numbers are well defined in $W$ and $\widetilde{W}$. Let $a, b$ be two closed curves in $W$. Then $\operatorname{lk}_{W^{\prime}}(a, b)=\sum_{\tau \in G} \mathrm{l}_{\widetilde{W}^{\prime}}(\tau a, b)$. We have

$$
\begin{aligned}
\beta(L) & =\operatorname{lk}_{S^{3}}\left(c_{1}, c_{1}^{+}\right)=\mathrm{lk}_{S^{3}}\left(c_{1}, \gamma\right) \\
& =\operatorname{lk}\left(c_{1}^{0}, \gamma^{0}\right)+\operatorname{lk}\left(c_{1}^{0}, \gamma_{1}\right)=\operatorname{lk}\left(c_{1}^{0}, \gamma^{0}\right)+\operatorname{lk}\left(c_{1}^{0}, c_{1}^{1}\right) .
\end{aligned}
$$

So

$$
\begin{aligned}
\operatorname{lk}\left(Y^{0}, Y^{1}\right) & =2 \operatorname{lk}\left(c_{1}^{0}, c_{1}^{1}\right)-2 \operatorname{lk}\left(\gamma^{0}, c_{1}^{0}\right) \\
& =2 \operatorname{lk}\left(c_{1}^{0}, c_{1}^{1}\right)-2 \operatorname{lk}\left(\gamma^{0}, c_{1}^{0}\right)-2 \operatorname{lk}\left(c_{1}^{0}, c_{1}^{1}\right)+2 \operatorname{lk}\left(c_{1}^{0}, c_{1}^{1}\right) \\
& =4 \operatorname{lk}\left(c_{1}^{0}, c_{1}^{1}\right)-2\left[\operatorname{lk}\left(\gamma^{0}, c_{1}^{0}\right)+\operatorname{lk}\left(c_{1}^{0}, c_{1}^{1}\right)\right] \\
& =4 \operatorname{lk}\left(c_{1}^{0}, c_{1}^{1}\right)-2 \beta(L),
\end{aligned}
$$

and

$$
\operatorname{lk}\left(Y^{0}, Y^{1}\right)=-2 \beta(L)+4 \operatorname{lk}\left(c_{1}^{0}, c_{1}^{1}\right) .
$$


The above equation is a recursive one. Consider the link $L_{1}=X \cup c_{1}$ a first derived link of $L$. By (2)

$$
\operatorname{lk}\left(c_{1}^{0}, c_{1}^{1}\right)=-2 \beta_{1}(L)+4 \mathrm{lk}\left(c_{2}^{0}, c_{2}^{1}\right)
$$

where $L_{2}=X \cup c_{2}$ is a second derived link of $L$. Thus,

$$
\begin{aligned}
\operatorname{lk}\left(Y^{0}, Y^{1}\right) & =-2 \beta(L)+4\left[-2 \beta_{1}(L)+4 \operatorname{lk}\left(c_{2}^{0}, c_{2}^{1}\right)\right] \\
& =-2 \beta(L)-2 \cdot 4 \beta_{1}(L)+4^{2} \operatorname{lk}\left(c_{1}^{0}, c_{2}^{1}\right) .
\end{aligned}
$$

In general $\operatorname{lk}\left(c_{j}^{0}, c_{j}^{1}\right)=-2 \beta_{j}(L)+4 \operatorname{lk}\left(c_{j+1}^{0}, c_{j+1}^{1}\right)$, so we have

$$
\begin{aligned}
\operatorname{lk}\left(Y^{0}, Y^{1}\right)= & -2 \beta_{0}(L)-2 \cdot 4 \beta_{1}(L)-2 \cdot 4^{2} \beta_{2}(L) \\
& -2 \cdot 4^{3} \beta_{3}(L)-\cdots+4^{N} \operatorname{lk}\left(c_{N}^{0}, c_{N}^{1}\right)
\end{aligned}
$$

or

$$
-\frac{1}{2} \operatorname{lk}\left(Y^{0}, Y^{1}\right)=\sum_{j=0}^{N-1} \beta_{j}(L)-2 \cdot 4^{N-1} \operatorname{lk}\left(c_{N}^{0}, c_{N}^{1}\right) \text {. }
$$

\section{REFERENCES}

[A] C. Arf, Untersuchungen über quadratische Formen in Körpern der Charackteristik 2, Crelles Math. J. 183 (1941).

[C] T. Cochran, Concordance invariants of coefficients of Conway's link polynomial, Invent. Math. 82 (1985), 527-41.

[Co] D. Cooper, The universal Abelian cover of a link (R. Brown and T. L. Thickstun, eds.), Lecture Notes Ser., vol. 48, London Math. Soc., 1979.

[KM] M. Kervaire and J. Milnor, Groups of homotopy spheres, Ann. of Math. 77 (1963).

[L] J. Levine, Polynomial invariants of knots of codimension two, Ann. of Math. 84 (1966).

[M] K. Murasugi, On the Arf invariant of links, preprint.

[R] R. Robertello, An invariant of knot cobordism, Comm. Pure Appl. Math. 18 (1965).

[S] R. Sato, Corbodisms of semi-boundary links, preprint.

Department of Decision Sciences, Concordia University, 1455 De Maisonneuve Boulevard West, Montreal, Quebec H3G IM8, Canada 